\title{
Stellar Clusters in the Outermost Milky Way Galaxy
}

\author{
J.E. Hesser, P.B. Stetson, S. van den Bergh
}

National Research Council of Canada, Herzberg Inst. of Astrophysics, Dominion Astrophysical Obs., Victoria, BC V8X 4M6, Canada

M. Bolte, J.A. Johnson

Lick Observatory, Santa Cruz, CA, USA

W.E. Harris

McMaster University, Hamilton, ON, Canada

D.A. VandenBerg

University of Victoria, Victoria, BC, Canada

R.A. Bell

University of Maryland, College Park, MD, USA

H.E. Bond, L.E. Fullton

STScI, Baltimore, MD, USA

G.G. Fahlman, H.B. Richer

University of British Columbia, Vancouver, BC, Canada

\begin{abstract}
HST $V, I$ color-magnitude diagrams (CMDs) of four outerhalo clusters, NGC 2419, Pal 3, Pal 4 and Eridanus, provide insight into the relative ages of old star clusters throughout the $200 \mathrm{kpc}$ diameter volume sampled, and thus into the formation epoch of the Milky Way galaxy.
\end{abstract}

\section{Introduction}

The ages, chemistry and other properties of outer Galactic halo star clusters make them powerful objects for comparison with the comparably remote dwarf spheroidal galaxies (dSphs), the oldest star clusters of the Magellanic Clouds and other Local Group galaxies. By constraining the chronology and duration of early cluster formation, they contribute to understanding how large galaxies like the Milky Way form, e.g.: 
Did the Galactic halo form from rapid collapse (Eggen et al. 1962; Sandage 1990), multi-Gigayear accumulation of many fragments (Searle \& Zinn 1978), or a combination of such processes? halo?

Did these processes occur in differing proportions in distinct regions of the

Are the more remote globular clusters with unusually red horizontal branches (HBs) for their $[\mathrm{Fe} / \mathrm{H}]$ - the "second-parameter" clusters - systematically younger (e.g., Searle \& Zinn; Lee et al. 1990, 1994; Chaboyer et al. 1996) than inner-halo objects, where the second-parameter phenomenon is weaker?

Inferred younger ages in the literature for clusters of the outer halo rely heavily upon HB morphology, which is sensitive to many parameters besides age. Truly direct age measurements that are capable of sharp tests of these scenarios can be made only with color-magnitude diagram (CMD) photometry reaching well below the main sequence turnoff (MSTO).

\section{New Data}

WFPC2 F555W (' $V$ ') and F814W (' $I$ ') photometry cleanly defines CMDs for the following clusters from their giant and HBs down through their upper main sequences: Pal $3\left(M_{V}=-5.2, \mathrm{R}_{\odot} \sim 87 \mathrm{kpc},[\mathrm{Fe} / \mathrm{H}] \sim-1.6\right) ; \mathrm{Pal} 4\left(M_{V}=-5.8\right.$, $\left.\mathrm{R}_{\odot} \sim 98 \mathrm{kpc},[\mathrm{Fe} / \mathrm{H}] \sim-1.3\right)$; Eridanus $\left(M_{V}=-4.8, \mathrm{R}_{\odot} \sim 78 \mathrm{kpc},[\mathrm{Fe} / \mathrm{H}] \sim\right.$ $-1.4)$; and NGC $2419\left(M_{V}=-9.5, \mathrm{R}_{\odot} \sim 88 \mathrm{kpc},[\mathrm{Fe} / \mathrm{H}] \sim-2.1\right)$.

Long and short exposures were obtained with WFPC2 (Cycles 4 \& 5). The sub-pixel-shifted images were combined to generate a master list of detected stars and to eliminate artifacts (cosmic rays and bad pixels), and then processed through the suite of DAOPHOT/ALLSTAR/ALLFRAME codes (Stetson 1994) to obtain the photometry. Calibration was carried out by a combination of new ground-based photometry in the imaged fields, plus the standard transformations derived by Holtzman et al. (1995).

CMDs for the four outer halo clusters typically reach $V_{\text {lim }}=27.0$ and cover more than three magnitudes of the main sequence. Each cluster exhibits a population of blue stragglers. Pal 3 contains several RR Lyrae variables.

\section{Relative Ages}

Accurate knowledge of abundances, and abundance ratios, is essential for both relative and absolute age determinations. Unfortunately, even in nearby Galactic globular clusters (whose giants are sufficiently bright for high-dispersion analyses), there is considerable dispersion among studies. For the outer-halo objects, abundance information is qualitatively different, as it is based upon lowdispersion spectra (often only of the calcium triplet in a few giants) and various indirect photometric indicators. To date our interpretations have focussed on relative ages determined by a number of differential fits between CMDs, which have been quantified by employing theoretical isochrones as interpolating tools. 


\subsection{Metal-Poor Clusters}

As shown by Harris et al. (1997), NGC 2419 and the nearby cluster M92 have indistinguishable ages, providing there are no unusual abundance ratios (e.g., $[\alpha / \mathrm{Fe}]$ ) in either of these $[\mathrm{Fe} / \mathrm{H}]=-2.2$ clusters. Thus, the earliest star (or globular cluster) formation began at essentially the same time everywhere in the Galactic halo throughout a region now almost $200 \mathrm{kpc}$ in diameter that handily encompasses the Magellanic Clouds.

\subsection{Intermediate-Metallicity Clusters}

To judge relative ages, we (Stetson et al. 1999) have compared the three secondparameter clusters via two standard ways of registering CMDs, $\Delta(V-I)$ and $\Delta V_{\mathrm{TO}}^{\mathrm{HB}}$. We have further used isochrones (VandenBerg et al. 1999) to predict, in a relative sense, the dependence of $\Delta V_{\mathrm{TO}}^{\mathrm{HB}}$ and $\Delta(V-I)$ on age, metallicity and other parameters. We have performed the same analysis on inner-halo clusters with abundances that are believed to bracket those of the outer-halo clusters. A consistent interpretation of the comparisons is:

a) Pal 3 is some 2 Gyr younger than M3, and Pal 4 about 1.5 Gyr younger than M5 if, in both cases, the compared clusters have identical abundances.

b) Eridanus could be either about 1 Gyr younger than Pal 4, or they could be the same age if Eridanus is some 0.2 dex more metal poor than Pal 4 . Since the available data suggest that the latter may be the case, the evidence for an age difference is tenuous.

c) Even though Pal 3 and Pal 4 appear indistinguishable in the differential age plot, Pal 3 is likely to be a Gyr older than Pal 4 because of its lower metal abundance. Similarly, because M5 is almost certainly more metal rich than M3, it is probably of order 1 Gyr younger.

\section{Concluding Remarks}

The two age indicators, $\Delta V_{\mathrm{TO}}^{\mathrm{HB}}$ and $\Delta(V-I)$, suggest that all three secondparameter clusters are younger than their nearby counterparts by 1.5 to $2 \mathrm{Gyr}$, which is in the same sense and approximately the same size predicted by $\mathrm{HB}$ star modelling (Lee et al. 1994, Lee 1992). Pal 14 (Sarajedini 1997) seems to be in the same age range.

However, if we ask what other parameters would have to be different for the "second parameter" clusters to have the same ages as the inner halo clusters, we find that an overestimation of the former's abundances by only 0.3 dex (either in $[\mathrm{Fe} / \mathrm{H}]$ or $[\alpha / \mathrm{Fe}])$ would be required. If Pal 3, Pal 4 and Eri were to have primordial abundances of $[\alpha / \mathrm{Fe}] \sim 0.0$, as Brown et al. (1997) found for Rup 106 and $\mathrm{Pal} 12$, that would erase half of the inferred age difference between them and the inner halo clusters.

Interestingly, recent HST work (Olsen et al. 1998, Johnson et al. 1998) suggests that, within CMD observational errors, the oldest LMC clusters do not appear to differ by more than a Gyr in age from the oldest Galactic globulars of comparable metallicity. This implies that the first major burst of star (cluster) formation occurred simultaneously in the two galaxies. 
While clear progress is being made towards achieving an accurate relative formation chronology for old stellar clusters in the Galactic halo and Magellanic Clouds from HST (or other) CMDs, we caution that the true answer may only be revealed when better relative abundance information is available for the clusters being compared.

\section{References}

Brown, J.A., Wallerstein, G., Zucker, D. 1997, A.J., 114, 180

Chaboyer, B., Demarque, P., Sarajedini, A. 1996, ApJ, 459, 558

Eggen, O.J., Lynden-Bell, D., Sandage, A. 1962, ApJ, 136, 748

Harris, et al. 1997, AJ, 114, 1030

Holtzman, J.A., et al. 1995, PASP, 107, 1065

Johnson, J.A., Bolte, M., Bond, H.E., Hesser, J.E., de Oliveira, C.M., Richer, H.B., Stetson, P.B., VandenBerg, D.A. 1998, IAU Symp. 190, in press

Lee, Y.-W. 1992, AJ, 104, 1780

Lee, Y.-W., Demarque, P., Zinn, R. 1990, ApJ, 350, 155

Lee, Y.-W., Demarque, P., Zinn, R. 1994, ApJ, 423, 248

Olsen, K.A.G., Hodge, P.W., Mateo, M., Olszewskie, E.E., Schommer, R.A., Suntzeff, N.B., Walker, A.R. 1998, MNRAS, in press

Sandage, A. 1990, JRASC, 84, 70

Sarajedini, A. 1997, AJ, 113, 682

Searle, L., Zinn, R. 1978, ApJ, 225, 357

Stetson, P.B. 1994, PASP, 106, 250

Stetson, P.B., et al. 1999, A.J., in press

VandenBerg, D.A., et al. 1999, in preparation

\section{Discussion}

Cannon: Presumably it is actually easier today for us to get metallicities of the distant clusters, than it was 10 years ago to work on the nearby clusters, given the big advances in the power of astronomical instrumentation. Is it not easy to get the data you want?

Hesser: It remains very challenging. In the sparse low luminosity outer halo clusters there are few red giants and ambiguities regarding their evolutionary state (1st or 2nd ascent?). Much of our abundance information rests on the $\mathrm{Ca}$ triplet observed in 2 or 3 giants at low dispersion. As an $\alpha$-element, $\mathrm{Ca}$ is a questionable surrogate for $[\mathrm{Fe} / \mathrm{H}]$. While many studies demonstrate an increase of $0.3-0.5 \mathrm{dex}$ in $[\mathrm{Ca} / \mathrm{Fe}]$ in the halo, some recent measurements by Brown \& Wallerstein in two other low-luminosity clusters show lower, if any, enhancements. 